\title{
Pathobiology of Salmonella, intestinal microbiota, and the host innate immune response
}

\section{Renato Lima Santos*}

Departamento de Clínica e Cirurgia Veterinárias, Escola de Veterinária, Universidade Federal de Minas Gerais, Belo Horizonte, Brazil

\section{Edited by:}

Constantino López-Macías, Mexican

Social Security Institute, Mexico;

University of Oxford, UK

\section{Reviewed by:}

Luisa Cervantes-Barragan,

Washington University School of

Medicine, USA

Kevin Maloy, University of Oxford, UK

${ }^{*}$ Correspondence:

Renato Lima Santos, Departamento de Clínica e Cirurgia Veterinárias,

Escola de Veterinária, Universidade

Federal de Minas Gerais, Av. Antônio Carlos 6627, Belo Horizonte, Minas

Gerais 31270-901, Brazil

e-mail: rsantos@vet.ufmg.br
Salmonella is a relevant pathogen under a clinical and public health perspective. Therefore, there has been a significant scientific effort to learn about pathogenic determinants of this pathogen. The clinical relevance of the disease, associated with the molecular tools available to study Salmonella as well as suitable animal models for salmonellosis, have provided optimal conditions to drive the scientific community to generate a large expansion of our knowledge about the pathogenesis of Salmonella-induced enterocolitis that took place during the past two decades. This research effort has also generated a wealth of information on the host immune mechanisms that complements gaps in the fundamental research in this area. This review focus on how the interaction between Salmonella, the microbiota and intestinal innate immunity leads to disease manifestation. As a highly successful enteropathogen, Salmonella actively elicits a robust acute intestinal inflammatory response from the host, which could theoretically lead to the pathogen demise. However, Salmonella has evolved redundant molecular machineries that renders this pathogen highly adapted to the inflamed intestinal environment, in which Salmonella is capable of outcompete resident commensal organisms. The adaptation of Salmonella to the inflamed intestinal lumen associated with the massive inflammatory response that leads to diarrhea, generate perfect conditions for transmission of the pathogen. These conditions illustrate the complexity of the co-evolution and ecology of the pathogen, commensals, and the host.

Keywords: Salmonella, innate immunity, intestinal microbiota, inflammation, enteritis, symbiosis

\section{INTRODUCTION}

Salmonella infection or the disease associated with it, salmonellosis, is most often characterized by enteritis. However, host restricted serotypes tend to induce higher levels of bacteremia, while some human restricted serotypes cause a systemic disease with mild enteric symptoms. All infections in warm blooded animal species and humans are due to one single Salmonella species, namely Salmonella enterica subsp. enterica, which includes more than 2,400 serotypes (1). Currently, there is an effort to reclassify S. enterica according to genotypes (based on multilocus sequence typing - MSLT) rather that serotypes. MSLT may be more accurate for predicting pathogenicity and host preferences (2). Although human restricted serotypes (i.e., Typhi and Paratyphi) cause a systemic disease named typhoid fever, several other serotypes, so-called "non-typhoidal Salmonella" (NTS) are capable of infecting human patients causing primarily an enteric disease characterized by enteritis and diarrhea. Most of the studies on Salmonella enteropathogenesis have been performed with serotype Typhimurium, therefore, unless stated otherwise, this review refers to Salmonella typhimurium.

Our understanding of the pathogenic mechanisms of NTS has markedly advanced over the past 20 years. Two important steps were crucial for achieving such advancement: (i) genetic manipulation of the pathogen that allowed researchers to dissect several of the Salmonella virulence factors, and (ii) development and characterization of suitable experimental models. Thus, the most significant molecular mechanisms employed by Salmonella for invasion and intracellular survival in host cells have been deciphered. Salmonella actively invades intestinal epithelial cells. The invasion process requires several effector proteins that are translocated through the Salmonella pathogenicity island-1 (SPI1)-encoded type III secretion system (TTSS) $(3,4)$. Salmonella is also capable of surviving intracellularly in phagocytic and nonphagocytic cells. Intracellular survival requires a second TTSS that is encoded by the Salmonella pathogenicity island 2 [SPI-2; $(4,5)]$.

In parallel to the progress in the field of molecular microbiology, experimental models, including epithelial, phagocytic, and other cell lines (6), as well as the development of animal models were instrumental for advancing in our knowledge on Salmonella enteropathogenesis $(7,8)$. Importantly, there are marked differences on how mammalian hosts respond to Salmonella (7). The mouse has been extensively used as a model for experimental infections. Importantly, marked differences in natural resistance has been demonstrated among mouse strains, which is associated with the resistant (e.g., strain 129sv) or susceptible (e.g., strains C57BL6/J and BALB/c) allele of the Slc11a1 (formerly known as Nramp1) gene (9). However, inoculation of mice with S. typhimurium results in a systemic infection that is not associated with diarrhea (7), but resembles typhoid fever caused by $S$. typhimurium in human patients (10). Therefore, aside of a few experimental reports with non-human primates $(11,12)$, bovine experimental infections became very relevant in this context (13) since cattle respond to NTS infection by developing an enteric disease that is clinically similar to human NTS infections $(13,14)$. 
Calves can be either orally infected (15) or subjected to surgical ligation of ileal loops that allow for a more precise assessment of early host responses (14). However, experimental studies performed in the 1980s have demonstrated that the absence of the intestinal microbiota has a profound impact on the outcome of infection in the mouse, rendering mice much more susceptible to infection (16). Furthermore, very early experimental studies have demonstrated that mice treated with streptomycin had an increased susceptibility to Salmonella (17), which allowed the development of a mouse model of Salmonella-induced typhlocolitis based on disruption of the intestinal microbiota by pre-treating the mice with streptomycin prior to challenge with S. typhimurium (18). This new model opened the opportunity to largely expand animal experimentation on Salmonella-induced intestinal inflammation, but it also clearly demonstrated the profound impact that the intestinal microbiota may have on the pattern of host response and outcome of infection.

The goal of this review is to discuss the advances in our knowledge on the innate intestinal immunity under the light shed by studies on the interaction between Salmonella, the intestinal microbiota, and the host.

\section{INTERDEPENDENCE OF THE INTESTINAL MICROBIOTA AND THE IMMUNE SYSTEM}

During the past few years, it has become increasingly clear that the intestinal microbiota plays a major role modulating intestinal mucosal immunity [reviewed by Ref. (19)]. Mammals coevolved with a complex population of commensal microorganisms that establish a mutually beneficial relationship to an extent that mammalians host more than $10^{14}$ microorganisms in the intestine (19). The significance of the microbiota for the development of the immune system is illustrated by the several immune defects that are observed in germ free mice, including decreased gut-associated lymphoid tissue, smaller mesenteric lymph nodes, and decreased antibody production, among other structural and functional deficiencies (19). It has been demonstrated that the host specific microbiota is required for full development of the mucosal immunity in the mouse (20). The Th-17 subset of T-cells is required for homeostasis and mucosal integrity, whereas the development of this cell population in the intestine requires the establishment of the microbiota, since germ free mice fail to develop Th-17 in the intestine (21). In a healthy individual, the microbiota prevents translocation of pathogenic microorganisms to the mesenteric lymph node thus preventing an undesirable immune response (22). Disruption of the microbiota (known as dysbiosis) due to antibiotic treatment favors translocation of even a non-invasive mutant $S$. typhimurium strain by phagocytes to the mesenteric lymph node (22).

In the past few years, a large number of relevant scientific reports have clearly established how the pathogen-associated molecular patterns (PAMPs) are recognized by their hosts (ranging from insects to mammalians) through pathogen recognition receptors (PRRs). However, a more recent wave of experimental evidences support the notion that molecules derived from the commensal microbiota are constantly sensed by host PRRs, which is a key step in establishing homeostasis [reviewed by Ref. (23)]. MyD88, a key adaptor protein for most TLRs (toll-like receptors), has been shown to play an important role in this context, since mice lacking MyD88 have a 100-fold increase in the number of bacteria associated with the intestinal mucosa (24). Therefore, considering that commensal microbiota is also sensed by PRRs, the term MAMPs, which stands for microbe-associated molecular patterns, has been proposed (25). Divergence between a PRR-mediated inflammatory response and PRR-mediated immune modulation and homeostasis is dependent on the concurrent presence of additional signals such as stimulation of cytosolic receptors by MAMPs (26). Importantly, in addition to sensing MAMPs, some of the cytosolic PRRs [i.e., Nod-like receptors (NLRs)] are capable of sensing signals associated with cell stress and damage, such as potassium influx, reactive oxygen species, membrane damage, etc. These signals are named danger-associated molecular patterns (DAMPs). Therefore, concomitant stimulation of extracellular PRRs and cytosolic PRRs by MAMPs or DAMPs allows the innate immune system to differentiate between stimuli from the commensal microbiota leading to homeostasis or pathogen triggered responses that lead to inflammation [reviewed by Ref. (27)].

While the establishment of the intestinal microbiota is a key event for immune maturation, conversely, immune cells in the intestine play an active role in shaping the composition of the microbiota, leading to homeostasis [reviewed by Ref. (28)]. For instance, the absence of CD4 ${ }^{+} \mathrm{T}_{\text {reg }}$ cells results in an unregulated $\mathrm{T}$-cell response against antigens from the microbiota, which causes intestinal inflammation (29). Mucosal antibodies, i.e., secretory IgA, also play a central role in shaping the microbiota. Impaired production of high affinity secretory IgA in the intestinal mucosa results in dysbiosis (30). Another very important component of this interaction between the host and microbiota are the intestinal epithelial cells (i.e., enterocytes, goblet cells, and Paneth cells). In addition to a physical barrier, structured by tight junctions between these cells that completely separate the apical from the basolateral compartment, the epithelium generates important factors that modulates expansion and composition of the microbiota. Goblet cells produce large amount of mucous that is a key element in homeostasis, while other cell types, particularly Paneth cells, generate antimicrobial peptides (31).

Interestingly, the influence of the microbiota is not restricted to the intestinal mucosal immunity, but it also impacts systemic immune sites. Antibiotic-induced dysbiosis results in impaired immune response against the influenza virus, while under these circumstances immunity is restored by rectal administration of PPR ligands, indicating that exposure of the intestinal mucosa to MAMPs is critical to modulating immunity (32). Indeed, there are experimental evidences of translocation of MAMPs from the intestine to systemic sites, where it modulates immune maturation, which indicates that the immune modulator role of the intestinal microbiota is not restricted to local tissues, influencing other distant immune organs (33). Interaction of the microbiota with the immune system is extremely complex, to the point that the microbiota may either favor or prevent the development of autoimmune disorders (34) as well as cancer development (35). Furthermore, the microbiota influences numerous other pleiotropic effects, both on pathologic events such as asthma, arthritis, inflammatory bowel diseases, obesity, and cardiovascular disease, as well as on physiological functions including organ morphogenesis, 
intestinal vascularization, tissue regeneration, bone homeostasis, metabolism, and behavior (36).

\section{SALMONELLA INTERACTION WITH THE INTESTINAL MICROBIOTA}

As previously mentioned, earlier studies have clearly demonstrated that disruption of the intestinal microbiota by treating mice with streptomycin results in increased susceptibility to Salmonella infection (17). Furthermore, the intestinal microbiota has a protective effect against Salmonella infection in the mouse (16). These studies prompted Barthel et al. (18) to develop a very useful experimental model based on treatment of mice with streptomycin followed by challenge with $S$. typhimurium. This model has been extensively utilized by the entire field, since experimental infections were previously largely restricted to more expensive and labor intensive animal models such as oral infections in calves (15) or the bovine ligated ileal loops (14). Further studies demonstrated that Salmonella elicits an inflammatory response in streptomycintreated mice that is pretty similar to that observed in Salmonellainfected germ free mice (37). While S. typhimurium infection in cattle triggers an acute inflammatory response that is characterized by massive infiltration of neutrophils (Figure 1) associated with variable degrees of necrosis, hemorrhage, erosion, and fibrinous pseudomembrane formation over the intestinal mucosa, particularly at the ileal Peyer's patches $(14,15)$, the same pathogen in the mouse does not elicit significant neutrophilic infiltration in the intestinal mucosa (7). Mice respond to S. typhimurium infection with a mild histiocytic infiltration, but in contrast they develop a marked systemic infection that is associated with lesions in the liver and spleen in the absence of diarrhea. Therefore, the development of the streptomycin-treated mouse model largely broadened the possibilities for in vivo experimental study of salmonellosis, allowing a marked worldwide expansion of animal experiments among several groups as well as genetic manipulation not just of the pathogen, but also of the host. Pretreatment with streptomycin results in a severe acute inflammatory response of the intestinal mucosa in response to S. typhimurium infection (Figure 2) (18). Although the original study that described this model demonstrated that streptomycin-treated mice have a much more efficient intestinal colonization with S. typhimurium (18), which suggests that the mechanism is likely due to lack of competition with components of the microbiota, this did not prove any direct cause or effect relationship between composition of the microbiota and the intrinsic nature of the innate intestinal immune response. Therefore, this model opened another extremely important area of investigation in this field, i.e., the role of the microbiota in the pathogenesis of NTS-induced enterocolitis.

Clinical treatment of human patients with antibiotics is recognized as a risk factor for subsequent Salmonella infection (38), which correlates well with what we have learned from the streptomycin-treated mouse model of Salmonella infection (18). However, the interaction of Salmonella with the microbiota is complex, and under certain circumstances pathogen and commensal may not necessarily have a mutually excluding relationship. For instance, a recent study demonstrated that carbohydrates metabolized by commensal microorganisms may serve as energy source for Salmonella. In that study, Bacteroides thetaiotaomicron,
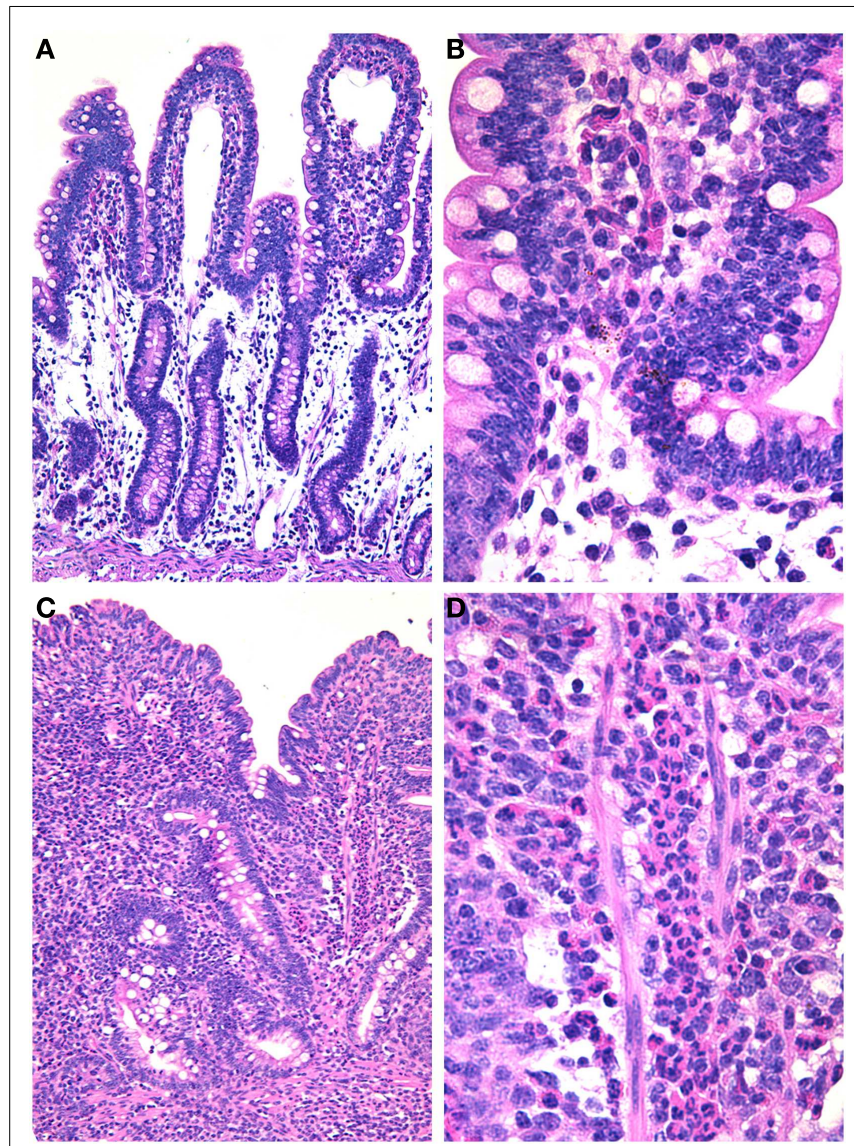

FIGURE 1 | Salmonella-induced enteritis in experimentally infected ligated ileal loops in calves. (A) Uninfected loop with no inflammatory reaction; $10 \times$ objective. (B) Higher magnification of uninfected loop; $40 \times$ objective. (C) Salmonella-infected loop with a severe and diffuse inflammatory infiltrate and blunting of the villi; $10 \times$ objective. (D) Higher magnification showing a diffuse and severe infiltration of neutrophils; $40 x$ objective. Hematoxylin and eosin.

which encodes sialidase that is required to release sialic acid from glycoconjugates, but does not have the enzymatic machinery to utilize sialic acid as a carbon source, generates free sialic acid, whereas S. typhimurium that lacks sialidase is capable of catabolizing this carbohydrate (39). S. typhimurium can also metabolize fucose generated in a similar manner. Therefore, members of the commensal microbiota are capable of releasing carbon sources that themselves cannot utilize, but that can be used as energy source by Salmonella (39). This process is thought to play a role in post antibiotic expansion of enteropathogens (39).

Susceptibility to different enteric pathogens is highly variable among different age groups. Interestingly, these differences in susceptibility may at least in part be related to changes in the composition of the intestinal microbiota [reviewed by Ref. (40)]. During early infancy the microbiota is highly dynamic, whereas in adults it is much more stable and composed predominantly by the phylum Bacteroidetes and Firmicutes (approximately 95\% of the microbiota), and elderly tend to have a predisposition to mild inflammation in the intestinal mucosa and decrease in the relative 


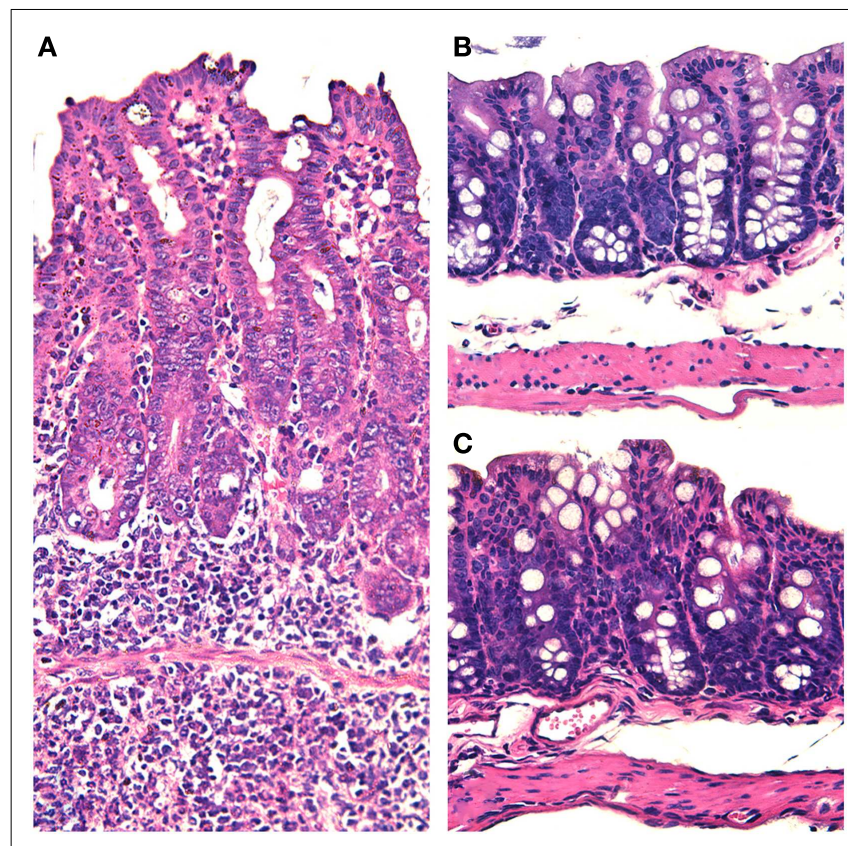

FIGURE 2 | Salmonella-induced typhlitis in mice with dysbiotic microbiota due to streptomycin treatment. (A) Marked thickening of the cecal wall with edema and increased cellularity due to a diffuse infiltration of inflammatory cells in a mouse with dysbiosis (pre-treated with streptomycin) and intragastrically infected with Salmonella enterica serotype Typhimurium. (B) Section of the cecum from a mouse intragastrically infected with Salmonella enterica serotype Typhimurium in the presence of a normal microbiota with no histopathological changes. (C) Section of the cecum from a healthy non-infected mouse. Note that all micrographs have the same magnification $20 \times$ objective. Hematoxylin and eosin.

abundance of Actinobacteria (essentially Bifidobacteria) and Firmicutes (40). Importantly, early and late stages of life, when the intestinal microbiota is less stable, with relatively lower numbers of Bacteroidetes and relatively higher numbers of gamma Proteobacteria, correspond to the period of higher susceptibility to some enteric pathogens (40).

With an elegant experimental approach, Chung et al. (20) demonstrated that germ free mice associated with human intestinal microbiota have increased susceptibility to Salmonella when compared to mice that had been associated with a normal mouse microbiota, indicating that under those experimental conditions the mouse microbiota is more protective against Salmonella than the human counterpart. This somewhat parallels the manifestation of Salmonella-induced intestinal pathology in these two host species, supporting the notion that the human microbiota may favor Salmonella-elicited intestinal inflammation, whereas the murine microbiota impairs the ability of Salmonella for triggering a host inflammatory reaction (7), which may be due to a lower antagonistic potential of the human microbiota when compared to that of mice.

An increasing number of experimental evidences points toward the notion that Salmonella has evolved multiple mechanisms by which it can overgrow members of the microbiota under conditions of an inflamed intestine (41). Several studies have identified
Salmonella effectors, among other bacterial factors, that play a role in triggering host inflammation in the intestine (42). Salmonella-induced enteropathogenesis is strongly associated with the ability of the pathogen to invade epithelial cells and the intestinal mucosa. Therefore, five effector proteins translocated through the (SPI-1)-encoded TTSS, namely SipA, SopA, SopB, SopD, and SopE2, are required for invasion and enteropathogenesis (43). Earlier studies have demonstrated that Salmonella has a competitive advantage over the microbiota in the inflamed intestine, whereas such advantage does not take place in the absence of inflammation (44). Quite a few mechanisms by which Salmonella takes advantage of intestinal inflammation have emerged recently. Lipocalin-2, a host antimicrobial peptide, is generated in the inflamed intestine in response to IL-17 and IL-22, whose production is triggered by Salmonella infection. This peptide prevents iron acquisition by intestinal microorganisms. It binds enterobactin, a siderophore produced by several enteric bacteria. However, Salmonella produces salmochelin (in addition to enterobactin), another siderophore that is not bound by lipocalin-2. Thus, under conditions of inflammation and abundance of lipocalin-2, Salmonella has a competitive advantage over other intestinal bacteria (45). Iron deprivation in the inflamed intestine induces expression of colicin Ib by Salmonella, which is a bacteriocin active against other Enterobacteriaceae, providing additional competitive advantage to Salmonella against part of the commensal microbiota under inflammatory conditions (46). Among other mechanisms by which Salmonella overgrow the commensal microbiota in the inflamed intestinal environment is based on its ability to acquire microelements, including zinc (47). In the inflamed intestine, calprotectin produced by neutrophils inhibits bacterial growth by sequestering zinc. However, Salmonella is capable of evading this host protective mechanism by expressing a high affinity zinc transporter named ZnuABC (47).

Another striking example of Salmonella adaptation to intestinal inflammation was provided by Winter et al. (48), who demonstrated that the inflamed intestinal environment provides a respiratory electron acceptor for Salmonella. Tetrathionate has been used as an enrichment medium for Salmonella isolation in vitro from samples containing competitive microbes since the 1920s. Reactive oxygen species generated during the inflammatory process triggered by Salmonella itself, oxidizes endogenous thiosulfate to generate tetrathionate, which can then be utilized as an anaerobic respiratory electron acceptor by Salmonella (48). This mechanism provides competitive advantage for Salmonella in the inflamed intestine while members of the microbiota perish due to environmental changes resulting from the massive Salmonellainduced inflammatory response. Salmonella-induced inflammation is associated with detachment of large numbers of enterocytes from the mucosa (14), Interestingly, ethanolamine derived from phosphatidylethanolamine, the most abundant phospholipid in membranes of detached enterocytes, can be utilized by Salmonella under anaerobic conditions using tetrathionate as electron acceptor in the inflamed gut (49). In addition to tetrathionate respiration, the effector protein SopE induces nitrate production by the host, which favors growth of Salmonella by allowing anaerobic nitrate respiration (50). Neutrophil-derived elastase, 
which is abundant in the inflamed intestine, suppresses components of the commensal microbiota, favoring intestinal growth of Salmonella (51). Mechanisms of Salmonella adaptation to the inflamed intestinal environment have been recently reviewed by Winter and Bäumler (52).

As a component of the innate host immune response, the inflammatory process should be seen as a host mechanism for preventing the spread of infection, which to some extent is completely correct, since in the absence of a neutrophilic response, Salmonella tends to spread more efficiently to systemic sites of infection, both in the mouse (53) as well as in cattle (54). These experimental observations parallel clinical disease since serotype Typhi that causes systemic infections does not elicit a significant intestinal neutrophilic response (10). However, as paradoxically as it may first seem, Salmonella evolved to take advantage of the host intestinal inflammatory response. Together, the studies discussed above clearly support the notion that Salmonella-induced inflammation is part of this pathogen strategy to create a highly favorable environment in the intestinal lumen for its own multiplication. However, Salmonella is a facultative intracellular pathogen, and that interaction with host cells is a determinant of the pathogenic capacity of this organism. Earlier studies strongly focused on the interaction of Salmonella with different host cell types, both in vitro and in vivo (6), missing a very important aspect of the big picture, which is the fact that only a fraction of the Salmonella population in a given host actually invades the mucosa during the acute phase of infection, while most of the organisms remain in the intestinal lumen (41). Excessive invasion of the intestinal mucosa by a larger fraction of the population of Salmonella could not be desirable under the pathogen point of view, since once within the host tissues, Salmonella is exposed to several efficient bactericidal mechanisms. This may explain the role of the SptP effector protein that reverses some of the molecular mechanisms used by Salmonella to invade intestinal epithelial cells (55).

Summarizing, Salmonella uses a kamikaze strategy based on a small fraction of its infecting population actively invading and triggering a massive acute inflammatory response. While this acute neutrophilic response may effectively restrict the infection mostly to enteric sites, largely preventing survival of invasive bacteria, and therefore preventing systemic dissemination of the pathogen, it also creates an intraluminal intestinal environment that favors the remaining larger fraction of the pathogen population that stays in the intestinal lumen, being able to multiply and effectively transmit the infection to the next host.

\section{MANIPULATION OF THE MICROBIOTA FOR PROPHYLACTIC AND THERAPEUTIC PURPOSES}

A thorough review of prophylactic and therapeutic approaches to modulate the function and/or composition of the microbiota is completely beyond the scope of this article. However, under a clinical point of view, it is relevant to point out some of the advances in this area. Clinical applications of probiotic and prebiotic has been recently reviewed by Vieira et al. (56). Probiotics are defined as live microorganisms which when administered in adequate amounts confer health benefits to the host [FAO/WHO, 2002 FAO/WHO Working Group, Guidelines for the Evaluation of Probiotics in
Food (2002). London, ON, Canada]. The notion of probiotic has been developed long time ago with the original observations of Metchnikoff in the beginning of the twentieth century, who identified microorganisms, particularly Bacillus bulgaricus (currently named Lactobacillus bulgaricus), which has beneficial effects on health and was the foundation of the yogurt industry (57). Probiotics, including different formulations and several different microorganisms in variable combinations, such as Saccharomyces boulardii, Bifidobacterium spp., Streptococcus thermophilus, Lactobacillus spp., Escherichia coli strain Nissle 1917, among several other microorganisms have been extensively used experimentally or therapeutically for treating enteric diseases with predominantly positive outcomes (56). However, particularly in immune compromised patients, the risk of sepsis should be taken in account when electing a probiotic therapeutic protocol (58). Prebiotics are food ingredients that are not digestible by the host and have favorable effects on specific components of the microbiota and intestinal homeostasis, although this concept may be expanded to include other food ingredients that do not completely fit the criteria for a prebiotic, but have similar effects, such as dietary fibers. Therapeutic or prophylactic combinations of probiotics and prebiotics are termed symbiotics (56).

A similar concept is linked to the ancient therapeutic practice of adoptive transfer of commensal microbiota from healthy individuals to patients with enteric diseases, particularly those associated with antibiotic therapy, which may be successful under certain conditions (59).

Specifically considering salmonellosis, there are experimental evidences indicating that probiotics may have a protective effect in mice experimentally challenged with Salmonella. Both germ free and conventional mice pre-treated with Saccharomyces cerevisiae UFMG 905 had lower levels of S. typhimurium dissemination upon experimental infection (60). Similarly, Lactobacillus acidophilus has protective effects against $S$. enteritidis infection in the mouse (61). Although it is not clear whether probiotics will ever have useful therapeutic applications in human patients infected with Salmonella, these experimental studies are relevant since probiotics and prebiotics have a significant potential for the animal industry, particularly for poultry and pigs. In food producing animal species, probiotics and prebiotics may prevent a high burden of Salmonella, thus mitigating the risk of transmission, with the additional significant benefit of decreasing the need and therefore the exposure of food producing animals to antibiotic treatment and growth promoters, which prevent emergence of antibiotic-resistant strains of pathogens. Indeed, several probiotics as well as food additives have been extensively studied under field conditions, but the results are highly variable, and strongly influenced by management, nutrition, environmental conditions, and obviously the levels of Salmonella challenge. Therefore, a general recommendation or a well-established protocol for probiotic or prebiotic prevention of Salmonella infection in farm animals is still unavailable $(62,63)$.

\section{CONCLUDING REMARIS AND PERSPECTIVES}

Since the first identification of microorganisms of the genus Salmonella in the beginning of the last century, a large body of knowledge has been accumulated regarding microbiological features of 
the organism, disease manifestation in different host species as well as its epidemiological implications. However, it was only during the last decade of the past century that molecular tools became available for dissecting pathogenic mechanisms of Salmonella. These molecular approaches preceded more sophisticated animal models, and therefore the pioneer investigations on Salmonella pathogenesis pictured a pathogen highly specialized in invasion and induction of a host response, as if the pathogen was indifferent to the myriad of commensal microorganisms in the intestinal environment. A subsequent wave of well-designed studies began to reveal, at a mechanistic level, some of the interactions between Salmonella and the microbiota in the intestine. Currently, it is clear that the complexity of these processes is unimaginable at this point so this is still a broadly open field for scientific investigation. A deeper knowledge of the pathobiology of Salmonella in the context of the intestinal environment may certainly open new perspectives for therapeutic approaches as well as for controlling animal and human salmonellosis.

\section{ACKNOWLEDGMENTS}

I thank Dr. Jacques R. Nicoli and Dr. Flaviano S. Martins for critically reviewing the manuscript, and Luciana F. Costa for technical assistance with the figure. Work in RLS lab is supported by CNPq (Conselho Nacional de Desenvolvimento Científico e Tecnológico, Brazil), FAPEMIG (Fundação de Amparo a Pesquisa do Estado de Minas Gerais, Brazil), and CAPES (Fundação de Aperfeiçoamento de Pessoal de Nível Superior, Brazil).

\section{REFERENCES}

1. Brenner FW, Villar RG, Angulo FJ, Tauxe R, Swaminathan B. Salmonella nomenclature. J Clin Microbiol (2000) 38:2466-7.

2. Achtman M, Wain J, Weill FX, Nair S, Zhou Z, Sangal V, et al. Multilocus sequence typing as a replacement for serotyping in Salmonella enterica. PLoS Pathog (2012) 8:e1002776. doi:10.1371/journal.ppat.1002776

3. Bajaj V, Lucas RL, Hwang C, Lee CA. Coordinated regulation of Salmonella typhimurium invasion genes by environmental factors is mediated by control of hilA expression. Mol Microbiol (1996) 22:703-14. doi:10.1046/j.1365-2958. 1996.d01-1718.x

4. Altier C. Genetic and environmental control of Salmonella invasion. J Microbiol (2005) 43:85-92.

5. Hansen-Wester I, Hensel M. Salmonella pathogenicity islands encoding type III secretion systems. Microbes Infect (2001) 3:549-59. doi:10.1016/S1286-4579(01) 01411-3

6. Santos RL, Bäumler AJ. Cell tropism of Salmonella enterica. Int J Med Microbiol (2004) 294:225-33. doi:10.1016/j.ijmm.2004.06.029

7. Santos RL, Zhang S, Tsolis RM, Kingsley RA, Adams LG, Bäumler AJ. Animal models of Salmonella infections: gastroenteritis vs. typhoid fever. Microbes Infect (2001) 3:1335-44. doi:10.1016/S1286-4579(01)01495-2

8. Kaiser P, Diard M, Stecher B, Hardt WD. The streptomycin mouse model for Salmonella diarrhea: functional analysis of the microbiota, the pathogen's virulence factors, and the host's mucosal immune response. Immunol Rev (2012) 245:56-83. doi:10.1111/j.1600-065X.2011.01070.x

9. Vidal SM, Pinner E, Lepage P, Gauthier S, Gros P. Natural resistance to intracellular infections: Nramp1 encodes a membrane phosphoglycoprotein absent in macrophages from susceptible (Nramp1 D169) mouse strains. J Immunol (1996) 157:3559-68.

10. Sprinz H, Gangarosa EJ, Williams M, Hornick RB, Woodward TE. Histopathology of the upper small intestines in typhoid fever. Biopsy study of experimental disease in man. Am J Dig Dis (1966) 11:615-24. doi:10.1007/BF02233509

11. Raffatellu M, Santos RL, Verhoeven DE, George MD, Wilson RP, Winter SE, et al. Simian immunodeficiency virus-induced mucosal interleukin-17 deficiency promotes Salmonella dissemination from the gut. Nat Med (2008) 14:421-8. doi:10.1038/nm1743
12. Santos RL, Almeida AP, Xavier MN, Paixão TA, Wilson RP, Dandekar S, et al. Enteric pathology and Salmonella-induced cell death in healthy and SIV-infected rhesus macaques. Vet Pathol (2011) 48:933-41. doi:10.1177/0300985810386468

13. Costa LF, Paixão TA, Tsolis RM, Baumler AJ, Santos RL. Salmonellosis in cattle: advantages of being an experimental model. Res Vet Sci (2012) 93:1-6. doi:10.1016/j.rvsc.2012.03.002

14. Santos RL, Zhang S, Tsolis RM, Bäumler AJ, Adams LG. Morphologic and molecular characterization of Salmonella typhimurium infection in neonatal calves. Vet Pathol (2002) 39:200-15. doi:10.1354/vp.39-2-200

15. Tsolis RM, Adams LG, Ficht TA, Bäumler AJ. Contribution of Salmonella typhimurium virulence factors to diarrheal disease in calves. Infect Immun (1999) 67:4879-85.

16. Nardi RM, Silva ME, Vieira EC, Bambirra EA, Nicoli JR. Intragastric infection of germfree and conventional mice with Salmonella typhimurium. Braz J Med Biol Res (1989) 22:1389-92.

17. Bohnhoff M, Drake BL, Miller CP. Effect of streptomycin on susceptibility of intestinal tract to experimental Salmonella infection. Proc Soc Exp Biol Med (1954) 86:132-7. doi:10.3181/00379727-86-21030

18. Barthel M, Hapfelmeier S, Quintanilla-Martínez L, Kremer M, Rohde M, Hogardt M, et al. Pretreatment of mice with streptomycin provides a Salmonella enterica serovar Typhimurium colitis model that allows analysis of both pathogen and host. Infect Immun (2003) 71:2839-58. doi:10.1128/IAI.71.5.2839-2858.2003

19. Round JL, Mazmanian SK. The gut microbiota shapes intestinal immune responses during health and disease. Nat Rev Immunol (2009) 9:313-23. doi:10.1038/nri2515

20. Chung H, Pamp SJ, Hill JA, Surana NK, Edelman SM, Troy EB, et al. Gut immune maturation depends on colonization with a host-specific microbiota. Cell (2012) 149:1578-93. doi:10.1016/j.cell.2012.04.037

21. Ivanov II, Frutos RL, Manel N, Yoshinaga K, Rifkin DB, Sartor RB, et al. Specific microbiota direct the differentiation of IL-17-producing T-helper cells in the mucosa of the small intestine. Cell Host Microbe (2008) 4:337-49. doi:10.1016/j.chom.2008.09.009

22. Diehl GE, Longman RS, Zhang JX, Breart B, Galan C, Cuesta A, et al. Microbiota restricts trafficking of bacteria to mesenteric lymph nodes by CX(3)CR1(hi) cells. Nature (2013) 494:116-20. doi:10.1038/nature11809

23. Chu H, Mazmanian SK. Innate immune recognition of the microbiota promotes host-microbial symbiosis. Nat Immunol (2013) 14:668-75. doi:10.1038/ni.2635

24. Cash HL, Whitham CV, Behrendt CL, Hooper LV. Symbiotic bacteria direct expression of an intestinal bactericidal lectin. Science (2006) 313:1126-30. doi:10.1126/science.1127119

25. Mackey D, McFall AJ. MAMPs and MIMPs: proposed classifications for inducers of innate immunity. Mol Microbiol (2006) 61:1365-71. doi:10.1111/j.13652958.2006.05311.x

26. Vance RE, Isberg RR, Portnoy DA. Patterns of pathogenesis: discrimination of pathogenic and nonpathogenic microbes by the innate immune system. Cell Host Microbe (2009) 6:10-21. doi:10.1016/j.chom.2009.06.007

27. Robertson SJ, Girardin SE. Nod-like receptors in intestinal host defense: controlling pathogens, the microbiota, or both? Curr Opin Gastroenterol (2013) 29:15-22. doi:10.1097/MOG.0b013e32835a68ea

28. Maynard CL, Elson CO, Hatton RD, Weaver CT. Reciprocal interactions of the intestinal microbiota and immune system. Nature (2012) 489:231-41. doi:10.1038/nature11551

29. Izcue A, Coombes JL, Powrie F. Regulatory lymphocytes and intestinal inflammation. Annu Rev Immunol (2009) 27:313-38. doi:10.1146/annurev.immunol. 021908.132657

30. Kawamoto S, Tran TH, Maruya M, Suzuki K, Doi Y, Tsutsui Y, et al. The inhibitory receptor PD-1 regulates IgA selection and bacterial composition in the gut. Science (2012) 336:485-9. doi:10.1126/science.1217718

31. Kurashima Y, Goto Y, Kiyono H. Mucosal innate immune cells regulate both gut homeostasis and intestinal inflammation. Eur J Immunol (2013) 43:3108-15. doi:10.1002/eji.201343782

32. Ichinohe T, Pang IK, Kumamoto Y, Peaper DR, Ho JH, Murray TS, et al. Microbiota regulates immune defense against respiratory tract influenza A virus infection. Proc Natl Acad Sci U S A (2011) 108:5354-9. doi:10.1073/pnas.1019378108

33. Clarke TB, Davis KM, Lysenko ES, Zhou AY, Yu Y, Weiser JN. Recognition of peptidoglycan from the microbiota by Nodl enhances systemic innate immunity. Nat Med (2010) 16:228-31. doi:10.1038/nm.2087

34. Chervonsky AV. Microbiota and autoimmunity. Cold Spring Harb Perspect Biol (2013) 5:a007294. doi:10.1101/cshperspect.a007294 
35. Cario E. Microbiota and innate immunity in intestinal inflammation and neoplasia. Curr Opin Gastroenterol (2013) 29:85-91. doi:10.1097/MOG. 0b013e32835a670e

36. Sommer F, Bäckhed F. The gut microbiota-masters of host development and physiology. Nat Rev Microbiol (2013) 11:227-38. doi:10.1038/nrmicro2974

37. Stecher B, Macpherson AJ, Hapfelmeier S, Kremer M, Stallmach T, Hardt WD. Comparison of Salmonella enterica serovar Typhimurium colitis in germfree mice and mice pretreated with streptomycin. Infect Immun (2005) 73:3228-41. doi:10.1128/IAI.73.6.3228-3241.2005

38. Pavia AT, Shipman LD, Wells JG, Puhr ND, Smith JD, McKinley TW, et al. Epidemiologic evidence that prior antimicrobial exposure decreases resistance to infection by antimicrobial-sensitive Salmonella. J Infect Dis (1990) 161:255-60. doi:10.1093/infdis/161.2.255

39. Ng KM, Ferreyra JA, Higginbottom SK, Lynch JB, Kashyap PC, Gopinath S, et al. Microbiota-liberated host sugars facilitate post-antibiotic expansion of enteric pathogens. Nature (2013) 502:96-9. doi:10.1038/nature12503

40. Kolling G, Wu M, Guerrant RL. Enteric pathogens through life stages. Front Cell Infect Microbiol (2012) 2:114. doi:10.3389/fcimb.2012.00114

41. Santos RL, Bevins CL, Adams LG, Raffatellu M, Tükel Ç, Tsolis RM, et al. Life in the inflamed intestine, Salmonella style. Trends Microbiol (2009) 17:498-506. doi:10.1016/j.tim.2009.08.008

42. Zhang S, Kingsley RA, Santos RL, Andrews-Polymenis H, Raffatellu M, Figueiredo J, et al. Molecular pathogenesis of Salmonella enterica serotype Typhimurium-induced diarrhea. Infect Immun (2003) 71:1-12. doi:10.1128/ IAI.71.1.1-12.2003

43. Zhang S, Santos RL, Tsolis RM, Stender S, Hardt WD, Bäumler AJ, et al. The Salmonella enterica serotype Typhimurium effector proteins SipA, SopA, SopB, SopD, and SopE2 act in concert to induce diarrhea in calves. Infect Immun (2002) 70:3843-55. doi:10.1128/IAI.70.7.3843-3855.2002

44. Stecher B, Robbiani R, Walker AW, Westendorf AM, Barthel M, Kremer M, et al. Salmonella enterica serovar Typhimurium exploits inflammation to compete with the intestinal microbiota. PLoS Biol (2007) 5:2177-89. doi:10.1371/journal. pbio.0050244

45. Raffatellu M, George MD, Akiyama Y, Hornsby MJ, Nuccio SP, Paixão TA, et al. Lipocalin-2 resistance confers an advantage to Salmonella enterica serotype Typhimurium for growth and survival in the inflamed intestine. Cell Host Microbe (2009) 5:476-86. doi:10.1016/j.chom.2009.03.011

46. Nedialkova LP, Denzler R, Koeppel MB, Diehl M, Ring D, Wille T, et al. Inflammation fuels colicin Ib-dependent competition of Salmonella serovar Typhimurium and E. coli in enterobacterial blooms. PLoS Pathog (2014) 10:e1003844. doi:10.1371/journal.ppat.1003844

47. Liu JZ, Jellbauer S, Poe AJ, Ton V, Pesciaroli M, Kehl-Fie TE, et al. Zinc sequestration by the neutrophil protein calprotectin enhances Salmonella growth in the inflamed gut. Cell Host Microbe (2012) 11:227-39. doi:10.1016/j.chom.2012. 01.017

48. Winter SE, Thiennimitr P, Winter MG, Butler BP, Huseby DL, Crawford RW, et al. Gut inflammation provides a respiratory electron acceptor for Salmonella. Nature (2010) 467:426-9. doi:10.1038/nature09415

49. Thiennimitr P, Winter SE, Winter MG, Xavier MN, Tolstikov V, Huseby DL, et al. Intestinal inflammation allows Salmonella to use ethanolamine to compete with the microbiota. Proc Natl Acad Sci U S A (2011) 108:17480-5. doi:10.1073/pnas.1107857108

50. Lopez CA, Winter SE, Rivera-Chávez F, Xavier MN, Poon V, Nuccio SP, et al. Phage-mediated acquisition of a type III secreted effector protein boosts growth of Salmonella by nitrate respiration. MBio (2012) 3:e00143-12. doi:10.1128/ mBio.00143-12

51. Gill N, Ferreira RBR, Antunes LCM, Willing BP, Sekirov I, Al-Zahrani F, et al. Neutrophil elastase alters the murine gut microbiota resulting in enhanced Salmonella colonization. PLoS One (2012) 7:e49646. doi:10.1371/journal.pone. 0049646
52. Winter SE, Bäumler AJ. Dysbiosis in the inflamed intestine: chance favors the prepared microbe. Gut Microbes (2014) 5:doi:10.4161/gmic.27129

53. Cheminay C, Chakravortty D, Hensel M. Role of neutrophils in murine salmonellosis. Infect Immun (2004) 72:468-77. doi:10.1128/IAI.72.1.468-477. 2004

54. Nunes JS, Lawhon SD, Rossetti CA, Khare S, Figueiredo JF, Gull T, et al. Morphologic and cytokine profile characterization of Salmonella enterica serovar Typhimurium infection in calves with bovine leukocyte adhesion deficiency. Vet Pathol (2010) 47:322-33. doi:10.1177/0300985809358037

55. Stebbins CE, Galán JE. Modulation of host signaling by a bacterial mimic: structure of the Salmonella effector SptP bound to Racl. Mol Cell (2000) 6:1449-60. doi:10.1016/S1097-2765(00)00141-6

56. Vieira AT, Teixeira MM, Martins FS. The role of probiotics and prebiotics in inducing gut immunity. Front Immunol (2013) 4:445. doi:10.3389/fimmu.2013. 00445

57. Podolsky SH. Cultural divergence: Elie Metchnikoff's Bacillus bulgaricus therapy and his underlying concept of health. Bull Hist Med (1998) 72:1-27. doi:10.1353/bhm.1998.0056

58. Theodorakopoulou M, Perros E, Giamarellos-Bourboulis EJ, Dimopoulos G. Controversies in the management of the critically ill: the role of probiotics. Int J Antimicrob Agents (2013) 42(Suppl):S41-4. doi:10.1016/j.ijantimicag.2013. 04.010

59. Buffie CG, Pamer EG. Microbiota-mediated colonization resistance against intestinal pathogens. Nat Rev Immunol (2013) 13:790-801. doi:10.1038/nri3535

60. Martins FS, Rodrigues AC, Tiago FC, Penna FJ, Rosa CA, Arantes RM, et al. Saccharomyces cerevisiae strain 905 reduces the translocation of Salmonella enterica serotype Typhimurium and stimulates the immune system in gnotobiotic and conventional mice. J Med Microbiol (2007) 56:352-9. doi:10.1099/jmm.0. 46525-0

61. Scapin D, Grando WF, Rossi EM, Perez KJ, Malheiros PS, Tondo EC. Antagonistic activity of Lactobacillus acidophilus LA10 against Salmonella enterica serovar enteritidis SE86 in mice. Braz J Microbiol (2013) 44:57-61. doi:10.1590/S151783822013005000024

62. Berge AC, Wierup M. Nutritional strategies to combat Salmonella in mono-gastric food animal production. Animal (2012) 6:557-64. doi:10.1017/ S1751731111002217

63. Kerr AK, Farrar AM, Waddell LA, Wilkins W, Wilhelm BJ, Bucher O, et al. A systematic review-meta-analysis and meta-regression on the effect of selected competitive exclusion products on Salmonella spp. prevalence and concentration in broiler chickens. Prev Vet Med (2013) 111:112-25. doi:10.1016/j.prevetmed. 2013.04.005

Conflict of Interest Statement: The author declares that the research was conducted in the absence of any commercial or financial relationships that could be construed as a potential conflict of interest.

Received: 27 March 2014; paper pending published: 30 April 2014; accepted: 13 May 2014; published online: 26 May 2014.

Citation: Santos RL (2014) Pathobiology of Salmonella, intestinal microbiota, and the host innate immune response. Front. Immunol. 5:252. doi: 10.3389/fimmu.2014.00252 This article was submitted to Microbial Immunology, a section of the journal Frontiers in Immunology.

Copyright $(0) 2014$ Santos. This is an open-access article distributed under the terms of the Creative Commons Attribution License (CC BY). The use, distribution or reproduction in other forums is permitted, provided the original author(s) or licensor are credited and that the original publication in this journal is cited, in accordance with accepted academic practice. No use, distribution or reproduction is permitted which does not comply with these terms. 